\title{
0 avanço da tecnociência médica e dos custos: um dilema bioético
}

\author{
The advancement of medical and costs technoscience: a bioethical dilemma
}

Antonio Pereira Filho*

\begin{abstract}
RESUMO: O avanço da tecnociência na saúde trouxe importante impacto econômico em todo o mundo, tanto pelo aumento dos custos na assistência à saúde quanto pelo aumento da população em faixas etárias cada vez mais elevadas. $\mathrm{O}$ artigo aborda a avaliação econômica das novas tecnologias em saúde nos seus aspectos de custo-benefício e custo-efetividade e como isso vem sendo feito no Brasil e em outros países. São analisadas as questões éticas que daí surgem, a partir do código de deontologia do profissional médico, considerando-se, no entanto, que o dilema ético vivido pelos demais profissionais da saúde não é muito diferente. A regulação das novas tecnologias tem por objetivo impedir gastos desnecessários por um lado para e para garantir o acesso de quem realmente precisa por outro lado. Conclui-se que a evolução da tecnociência em saúde e o estrondoso aumento dos custos assistenciais são um dos maiores dilemas bioéticos do século XXI, e equacionar os conceitos bioéticos milenares da medicina com os novos paradigmas da tecnociência e da gestão de saúde será uma árdua tarefa.
\end{abstract}

PALAVRAS-ChaVE: Análise Custo-Benefício. Gastos em Saúde. Bioética.

ABSTRACT: The advancement of technoscience in the health field caused an important economical impact in the entire world, both due to the increase of costs in health assistance and because of the increase of the population from older age groups. This article approaches the economical evaluation of the new technologies in health in their cost-benefit and cost-effectiveness aspects, as well as how has been done in Brazil and in other countries. We examine ethical questions emerging from these data considering deontology code of the medical professional, but also considering, however, that the ethical dilemma lived by other health professionals do not differ much from this one. The regulation of the new technologies aims to prevent unnecessary expenses on the one side and to guarantee the access by those who really need, on the other. We conclude that the evolution of technoscience in the health field and the exponential increase of assistance costs are two of the biggest bioethical dilemmas of the $21^{\text {st }}$ century; and to equate thousand-years-old bioethical concepts of medicine with the new paradigms of technoscience and health management will be a demanding task.

KEYWORDS: Cost-Benefit Analysis. Health Expenditures. Bioethics.

\section{INTRODUĈ̣̃O}

Nas últimas décadas, a medicina passou por transformações em virtude do avanço tecnológico. Progressivamente a tecnologia aperfeiçoa meios de diagnose e terapia levando a diagnósticos cada vez mais precoces e tratamentos clínicos e cirúrgicos em constante aprimoramento.

O resultado disso é a melhora na qualidade de vida e um aumento substancial em todo o mundo da expectativa de vida. Nos próximos anos, a barreira do centésimo aniversário será alcançada por boa parte da população mundial.

Esse cenário tem, entretanto, sérias consequências econômicas, não só no aumento de custos com assistência à saúde, mas também com aspectos previdenciários. Vários países já estão alterando a idade da aposentadoria em virtude da insolvência financeira dos sistemas previdenciários.

Por trás de todo esse avanço tecnológico existe todo um complexo industrial da saúde que tem uma lógica empresarial capitalista que nem sempre tem os mesmos interesses dos sistemas de saúde públicos ou privados, muito pelo contrário, os embates desses interesses são cada vez mais frequentes ${ }^{1}$.

O aumento de custos com assistência à saúde é um problema a ser enfrentado até pelos chamados países desenvolvidos. Que dizer do problema enfrentado pelo Brasil, onde a maioria da população depende exclusivamente do sistema único de saúde (SUS) notoriamente subfinanciado ${ }^{2}$, como veremos adiante?

Além disso, a base produtiva do complexo industrial da saúde no Brasil ainda é frágil, o que prejudica não só a prestação de serviços universal para a população, de 
acordo com a Constituição Federal, mas também uma inserção mais competitiva desse poderoso complexo industrial em um cenário econômico mundial globaliza$\mathrm{do}^{3}$.

O artigo aborda o inevitável tema da avaliação econômica das novas tecnologias em saúde nos seus aspectos de custo-benefício e custo-efetividade e como isso vem sendo feito no Brasil e outros países.

Novos paradigmas surgiram com o avanço da tecnologia na assistência à saúde, e esses paradigmas trouxeram novas questões éticas, até então inexistentes. Abordamos essas novas questôes éticas trazidas pela biotecnologia do ponto de vista da deontologia médica ${ }^{4}$.

\section{O COMPLEXO INDUSTRIAL DA SAÚDE}

O complexo industrial da saúde é um setor vital da economia mundial. Segundo dados da Organização Mundial da Saúde (OMS), os gastos nesse complexo envolveram, em 2007, 9,7\% do produto interno bruto (PIB) do planeta. Os Estados Unidos da América (EUA), segundo últimos dados, chegou a gastar 5,3 bilhões de dólares/ano, representando $15,7 \%$ do PIB. No Brasil, somando-se os setores públicos e privados, gasta-se $8,4 \%$ do PIB $^{5}$.

Uma série de fatores aumenta a expansão do setor, entre eles a maior conscientização de que é necessário cuidar mais e melhor da saúde, o surgimento de novas tecnologias, o aumento do poder aquisitivo e a longevidade da população. Nesse último quesito, dados do Instituto Brasileiro de Geografia e Estatística de 2009 dão conta que a expectativa de vida do brasileiro, ao nascer, é de 73,2 anos, tendo subido mais de dez anos entre 1980 e 2009. Estatística do mesmo instituto mostra um crescimento constante da população acima dos 60 anos, e essa população deve dobrar nos próximos 20 anos, atingindo $30 \%$ do total da população brasileira em $2050^{5}$. A consequência desse cenário é um previsível aumento de custos com assistência à saúde nos próximos anos.

Uma característica do setor saúde, que agrava ainda mais a situação, é que existem várias questões de gestão ainda não resolvidas e que não há consenso em como abordá-las. Muitos economistas são de opinião que o setor é o de maior complexibilidade de gestão na economia devido a: 1) Amplo escopo, desde promoção da saúde até tratamentos complexos de doenças; 2) consumo não desejado em caso de doença; 3) importante impacto na qualidade e duração da vida do consumidor; 4) por sua relevância social necessita da presença do Estado ou ao menos de sua regulação; 5) é um dos principais setores da economia de todos os países; 6) setor de conhecimento intensivo em constante evolução e de domínio desigual entre profissionais, consumidores, fontes pagadoras e industriais.

O avanço da biotecnologia e suas aplicações na prática médica tornou-se um novo paradigma na assistência à saúde. Podemos dividir as novas tecnologias em três grandes grupos: 1) tecnologias de suporte de vida (monitores, respiradores, aparelhos de diálise, marca-passo, etc.); 2) tecnologias diagnósticas (tomografia, ressonância, medicina nuclear, testes genéticos, etc.); 3) tecnologias cirúrgicas (próteses, stents, lentes intraoculares, cirurgias vídeo-laparoscópicas circulação extracorpórea, etc.).

Além desses três grandes grupos, surgiu, nos últimos anos, a tecnologia da transcendência (engenharia genética, uso de célula-tronco, clonagem), a nanotecnologia (cirurgias, diagnósticos, manipulação de moléculas) e a telemedicina (cirurgias e conferências a distância, prontuário eletrônico).

Todos esses avanços tecnológicos só se concretizarão graças ao complexo médico-industrial. Esse complexo tornou-se uma área de negócios atrativa por sua alta lucratividade, porém geradora de conflitos de interesse por outro lado.

As novas tecnologias têm em geral três tempos na sua evolução: 1) surpresa e encanto com seu potencial; 2) reconhecimento de limitaçôes, erros e riscos da nova tecnologia; 3) regulação, controle de riscos e análise do custo-benefíción .

$\mathrm{O}$ avanço da biotecnologia tem provocado uma discussão difícil: deve-se gastar com aparelhos e fármacos de última geração ou investir em tratamentos convencionais para as doenças mais comuns, combate à desnutrição, saneamento básico, doenças infectocontagiosas? E as novas tecnologias que evitam internações e tratamentos caros 
graças a um diagnóstico mais precoce? Esse dilema é vivenciado pelo governo e planos de saúde. Uma primeira decisão de não investir em uma nova tecnologia por ser cara pode ser uma decisão errada em médio e longo prazo, pois ela pode, na frente, trazer economia assistencial maior que o investimento inicial ${ }^{6,7}$.

Quando se analisa o complexo econômico-industrial da saúde, percebe-se a necessidade de uma análise mais sistemática das complexas relações de interesses entre produção, tecnologia e finalidade social. É necessário buscar um modelo de desenvolvimento para esse complexo industrial que seja socialmente inclusivo.

Esse complexo industrial da saúde é composto por subsistemas: indústria química, biotecnológica, mecânica, eletrônica, de materiais e de prestação de serviço que interagem entre si, conforme o Quadro 1:

Quadro 1. Complexo industrial da saúde

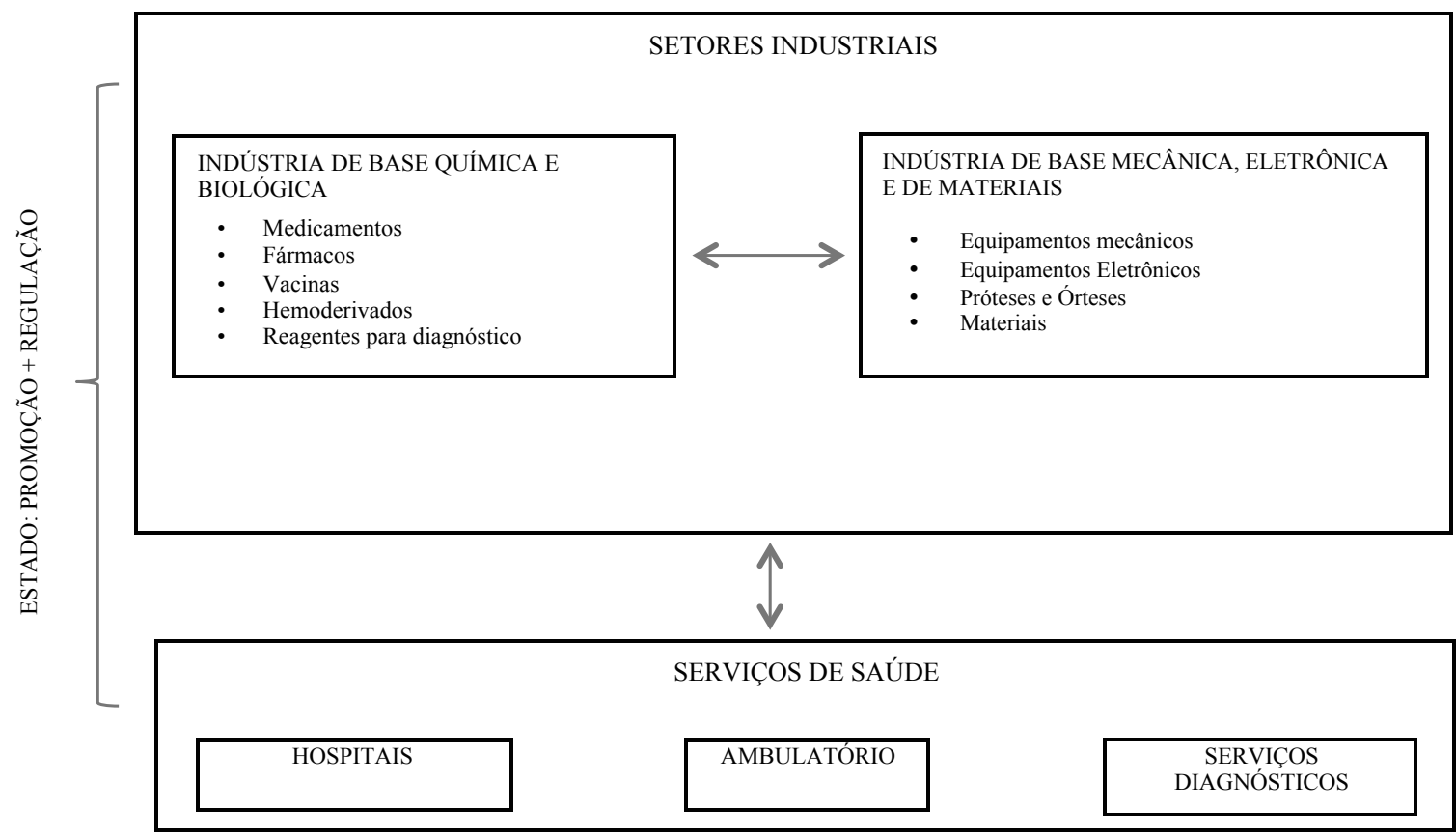

Fonte: Gadelha ${ }^{1}$.

Todos esses subsistemas apresentam elevado grau de potencial de inovação, o que os torna altamente competitivos. No Brasil, a indústria da saúde ainda é frágil na maior parte dos subsetores, o que leva a um grande déficit na balança comercial do setor devido ao grande volume das importações, o que cria obstáculos para oferta universal de bens e serviços, vulnerabilizando a política nacional de saúde. Daí a necessidade de criar uma política de desenvolvimento para esse setor que articule o econômico e o social, o que deve ter uma participação forte do Estado $^{8}$.
Como existe uma histórica desarticulação dos subsistemas no Brasil, o complexo produtivo de origem química, biológica, mecânica, eletrônica e de matérias desenvolvem-se sem considerar seu caráter sistêmico, isto é, sem levar em conta o subsistema de prestação de serviços, o que acaba por prejudicar, em um segundo momento, a produção e inovação.

O subsistema de base química e biotecnologia tem elevado grau de internacionalização e intensa concentração de mercado em poucas empresas, como ocorre com a indústria farmacêutica, um oligopólio. A exceção desse 
setor é o desenvolvimento nacional de produção de vacinas, que se deve a um programa de investimentos para atender o programa nacional de vacinação.

O mesmo precisaria ser feito com a indústria farmacêutica, em que a produção nacional é muito fraca e o oligopólio internacional ameaça a gestão da saúde coletiva brasileira. Os grandes laboratórios chegam a negligenciar doenças altamente prevalentes no Brasil, como chagas, leishmaniose, tuberculose, entre outras ${ }^{3}$.

A atuação do Estado é fundamental, como ocorreu com as vacinas e está ocorrendo com sangue e hemoderivados; estão sendo feitos investimentos na Hemobrás para que o País seja autossuficiente em 2014. Outra atuação importante que o Estado teve foi a quebra de patentes dos antirretrovirais.

O subsistema de base mecânica, eletrônica e de materiais é bastante variado, com equipamentos altamente sofisticados, como é o caso dos exames por imagem e equipamentos bastante simples de uso corriqueiro. A exemplo do subsistema de base química e biotecnológica, também existe um oligopólio marcadamente nos equipamentos de maior sofisticação. Por outro lado, empresas de menor porte dos países em desenvolvimento alcançaram bons resultados com a produção de equipamentos de uso cotidiano, tornando o subsistema heterogêneo.

O subsistema de base mecânica, eletrônica e de produtos tem características próprias na produção. A produção de novos equipamentos, cada vez mais sofisticados, não substituem os anteriores, mas a eles se adicionam, impactando fortemente os custos assistenciais na saúde.

Novamente o Estado necessita atuar como mediador nessa área política, entre os interesses do complexo econômico industrial da saúde e os interesses de uma assistência médica exequível para as populações dos países.

O Brasil pode estabelecer uma relação virtuosa entre a expansão da indústria de saúde e a implementação das políticas do SUS. Sem essa relação virtuosa, fica comprometido o atendimento às demandas sociais do SUS e ainda com grande aumento de gastos.
Finalmente, vamos abordar o subsistema de serviços, que é o de maior peso econômico no complexo industrial da saúde. Nesse setor está uma parcela importante do total da renda e empregos em nosso País.

Os serviços exercem um papel sistêmico nos outros subsistemas, na medida em que são ao mesmo tempo consumidor e demandante, influenciando o volume de produção de medicamentos, equipamentos, materiais, sangue e hemoderivados, etc. Os serviços são, assim, o elo que organiza toda a cadeia produtiva na saúde ${ }^{3}$.

O Estado brasileiro tem tido um papel importante na compra de serviços por meio do SUS, porém existe uma evidente incoerência entre o volume do financiamento público e os preceitos constitucionais em relação à saúde (a saúde é direito do cidadão e dever do Estado).

Esse subfinanciamento do SUS fez surgir um importante setor de serviços de assistência privada no seu conjunto denominado sistema supletivo de saúde (operadoras e planos de saúde), com práticas de mercado capitalistas muito semelhantes à indústria.

\section{IMPACTO ECONÔMICO DA TECNOLOGIA NO BRASIL}

A entrada ou saída de uma tecnologia no atendimento à saúde de uma população gera a necessidade da análise de impacto orçamentário (A.I.O.) para avaliação das consequências financeiras, uma vez que os recursos são sempre finitos.

É necessário fazer a distinção entre A.I.O. e a análise do custo efetividade (A.C.E.).

$\mathrm{Na}$ A.C.E., compara-se o efeito na relação de custos e ganhos decorrentes da substituição de uma tecnologia por outra, e, na A.I.O., é analisado o impacto econômico na introdução ou remoção de uma tecnologia considerando-se o conjunto das tecnologias disponíveis para o problema de saúde em análise. Para tanto, devem ser analisados os seguinte elementos ${ }^{7}$ : 1) o gasto atual com uma dada situação de saúde; 2) os candidatos à nova tecnologia; 3) os custos diretos da nova tecnologia; 4) o grau de inserção da nova tecnologia após a sua incorporação. 
Pode-se dizer que a A.I.O. é fundamental para gestão de qualquer sistema de saúde, pois sem ela não se faz previsão orçamentária.

A.I.O. e A.C.E. são complementares. Enquanto a A.C.E. estima para cada indivíduo quanto será gasto para que ele tenha um ano de vida a mais, a A.I.O. estima os gastos que a nova tecnologia trará para uma determinada população em um determinado intervalo de tempo ${ }^{7}$.

Quase todos os trabalhos sobre A.I.O. baseiam-se nas diretrizes da International Society for Pharmacoeconomics and Outcomes Research (ISPOR)'.

\section{Avaliação de Tecnologia em Saúde (ATS)}

Em função do alto custo das novas tecnologias e da necessidade política e ética de oferecer aos usuários, tanto do sistema público quanto do sistema privado, todo esse avanço, é que em todo o mundo se faz a avaliação de tecnologia em saúde, sendo isso fundamental em países em desenvolvimento, onde não há recursos para oferecer tudo para todos.

Vamos, neste artigo, citar rapidamente quais são os organismos que fazem a ATS no SUS e na medicina supletiva.

No âmbito do SUS, o Quadro 2 resume essas instâncias de regulação.

Quadro 2. Fluxo simplificado de incorporação de tecnologias no SUS

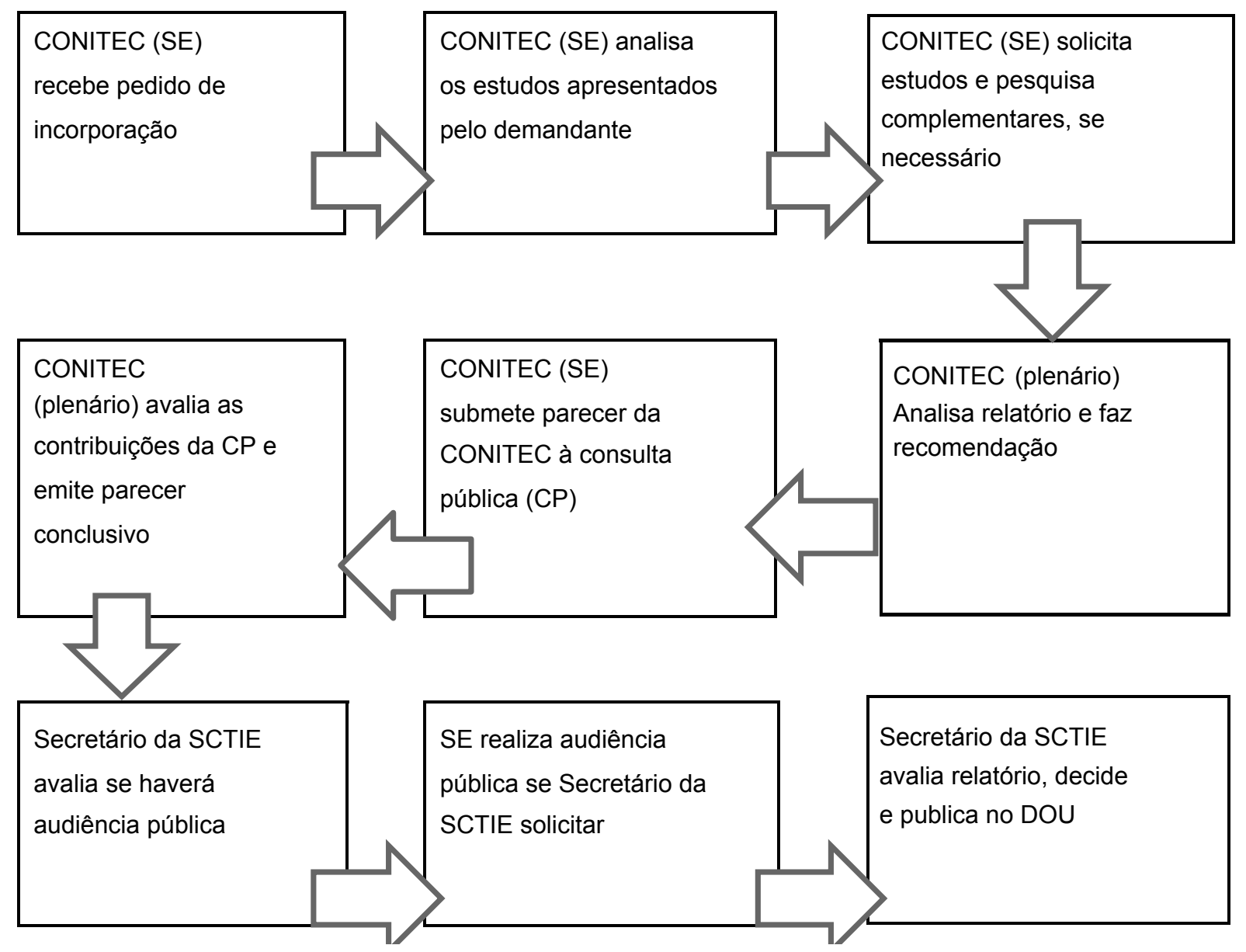

Legenda: SE - Secretaria Executiva; SCTIE - Secretaria de Ciência, Tecnologia e Insumos Estratégicos; DOU - Diário Oficial da União; CP Consulta Pública; CONITEC - Comissão Nacional de Incorporação de Tecnologias no SUS.

Fonte: Ministério da Saúde. Disponível em: http://portal.saude.gov.br/portal/arquivos/pdf/fluxosimplificadoCONITEC.pdf. Acesso 24 Abr 2013. 
No âmbito da saúde suplementar, a ATS ficou bastante fragmentada com o surgimento da ANS, que chamou para si a definição do rol de procedimentos a serem obrigatoriamente oferecidos aos usuários de plano de saúde. Esse rol passou a ser, na prática, o principal balizador das novas tecnologias a serem fornecidas. A ANVISA também exerce importante papel pelo seu poder regulador ao ser o órgão que aprova a utilização em território nacional de novas tecnologias. Por fim, como algumas operadoras de planos de saúde estão criando redes próprias de hospitais, laboratórios e ambulatórios, tais operadoras ganham alguma autonomia na incorporação de tecnologia que, até então, ficava exclusivamente na mão dos prestadores de serviços ${ }^{10}$.

\section{CONCLUSÃO: 0 DILEMA BIOÉTICO}

Vamos nos ater a questões éticas do profissional médico e ao seu código de deontologia, porém o dilema ético dos médicos não é muito diferente do vivido pelos demais profissionais da saúde quando se aborda o espinhoso tema da evolução da tecnociência em saúde e o incremento brutal de custos que ela carreia.

Em todos os códigos de ética dos profissionais da saúde, existe o compromisso de oferecer o melhor ao paciente sob seus cuidados e não deixar de envidar esforços para com a manutenção da vida e de sua qualidade.

Igualmente em todos os códigos, sem exceção, é ressaltada a autonomia do profissional em seus atos e suas escolhas em favor do paciente.

Destacam-se alguns artigos do código de ética médica vigente no Brasil desde 2010, por força da Resolução do Conselho Federal de Medicina 1931 ${ }^{11}$.

Princípios Fundamentais:

I - A medicina é uma profissão a serviço da saúde do ser humano e da coletividade e será exercida sem discriminação de nenhuma natureza.

II - O alvo de toda a atenção do médico é a saúde do ser humano, em benefício do qual deverá agir com o máximo de zelo e o melhor de sua capacidade profissional.

$\mathrm{V}$ - Compete ao médico aprimorar continuamente seus conhecimentos e usar o melhor do progresso científico em benefício do paciente.

XVI - Nenhuma disposição estatutária ou regimental de hospital ou instituição, pública ou privada, limitará a escolha, pelo médico, dos meios cientificamente reconhecidos (...).

Portanto, fica claro que, já nos princípios fundamentais, que são as "cláusulas pétreas" da deontologia médica, existe o compromisso ético que os médicos devem ter para com seus pacientes de não negar-lhes o acesso aos avanços da tecnociência médica.

Mas, além dos princípios fundamentais, dois outros artigos abordam o tema, senão vejamos:

Artigo 20: é vedado ao médico permitir que interesses pecuniários, políticos, religiosos ou de quaisquer outras ordens, do seu empregador público ou superior hierárquico ou do financiador público ou privado da assistência à saúde interfiram na escolha dos melhores meios de prevenção, diagnóstico ou tratamento disponíveis e cientificamente reconhecidos no interesse da saúde do paciente ou da sociedade.

Artigo 32: É vedado ao médico deixar de usar todos os meios disponíveis de diagnóstico e tratamento, cientificamente reconhecidos e a seu alcance, em favor do paciente.

Deve-se assinalar que o não cumprimento do artigo 32 está intimamente ligado, em praticamente todos os casos, à infração do artigo $1^{\circ}$ na modalidade negligência, porque é claro que quem não utilizou tudo que podia utilizar foi negligente.

É importante destacar que o Código de Ética Médica não nasceu agora. $\mathrm{Na}$ verdade, já na Grécia antiga o juramento de Hipócrates (400 a.C.) citava: "Aplicarei os regimes para o bem dos doentes segundo meu poder 
e entendimento (...)" e "Em toda casa, aí entrarei para o bem dos doentes (...)".

Portanto estamos diante de conceitos que se iniciaram cerca de 400 anos antes de Cristo e foram se aprimorando ao longo de toda a idade antiga, média e moderna em todo o mundo por meio de juramentos, códigos e declaraçôes internacionais. Sem dúvida isso explica por que esses princípios éticos são tão arraigados na profissão médica.

Os custos com materiais e medicamentos utilizados durante séculos no tratamento dos doentes não eram altos. A indústria voltada a instrumentos médicos era artesanal e sem grande importância econômica em todo o mundo.

A partir do final do século XIX e século XX, notadamente na sua segunda metade, é que ocorre um impressionante desenvolvimento da tecnologia voltada para a saúde, que impulsiona o surgimento de um poderoso complexo industrial do ponto de vista econômico.
Foi pouco tempo para os médicos perceberem que os preceitos éticos milenares, ainda bem explícitos nos códigos de ética médica de todos os países, necessitariam de adaptaçôes. A classe médica mundial assistiu perplexa e até indignada o surgimento de mecanismos regulatórios, graves problemas de financiamento, estudos de impacto orçamentário estabelecendo limites e de custo-efetividade ditando condutas.

Novos saberes e novas áreas de atuação surgiram, até 50 anos atrás inimagináveis, como a engenharia genética, a nanotecnologia, a telemedicina, a economia em saúde, a farmacoeconomia, o biodireito, só para citar alguns.

Não há mais dúvidas que a evolução da tecnociência em saúde e o estrondoso aumento dos custos assistenciais são um dos maiores dilemas bioéticos do século XXI para médicos e demais profissionais de saúde. Equacionar os conceitos bioéticos milenares da medicina com os novos paradigmas da tecnociência e da gestão de saúde será uma árdua tarefa que envolverá a todos que atuam na nobre arte de curar.

\section{REFERÊNCIAS}

1. Gadelha CAG. O Complexo industrial da saúde e a necessidade de um enfoque dinâmico na economia da saúde. Ciên Saúde Colet. 2003 [acesso 23 Maio 2013];8(2):521-35. Disponível em: http://www.scielo.br/scielo.php?pid=S1413-81232003000200015\&script=sci arttext. DOI: http://dx.doi.org/10.1590/S1413-81232003000200015.

2. Faveret ACSC. A vinculação constitucional de recursos para a saúde: avanços, entraves e perspectivas. Ciên Saúde Colet. 2003 [acesso 23 Maio 2013];8(2):371-8. Disponível em: http://www.scielo.br/scielo.php?pid=S1413-81232003000200004\&script=sci arttext. DOI: http://dx.doi.org/10.1590/S1413-81232003000200004.

3. Gadelha CAG, Costa LS, Maldonado J. O Complexo econômico-industrial da saúde e a dimensão social e econômica do desenvolvimento. Rev Saúde Pública. 2012 [acesso 23 Maio 2013];46 Supl 1. Disponível em: http://www.scielo.br/scielo.php?pid=S0034-89102012000700004\&script=sci arttext. DOI: http://dx.doi.org/10.1590/S0034-89102012005000065.

4. Antunes JL. Medicina e tecnologia. Janus. 2009 [acesso 23 Abr 2013];2. Disponível em: http://janusonline.pt/2009/2009 2 21.html.

5. Pedroso MC, Malik AM. As quatro dimensões competitivas da saúde. Harvard Business Rev. 1969 [acesso 23 Abr 2013]. Disponível em: http://www.hbrbr.com.br/materia/quatro-dimensoes-competitivas-da-saude.

6. Hume M. Saúde e os avanços da tecnologia. Rev Hospitais Brasil. [s. d.] [acesso 23 Abr 2013]. Disponível em: http://www.brasilmedicina.com.br/noticias/pgnoticias det.asp? Codigo=1442\&AreaSelect=1.

7. Silva ALF, Ribeiro RAR, Santos VCC, et al. Diretriz para análises de impacto orçamentário de tecnologias em saúde no Brasil. Cad Saúde Pública. 2012 [acesso 23 Abr 2013];28(7):1223-38. Disponível em: http://www.scielosp.org/scielo.php?script=sci arttext\&pid=S0102-311X2012000700002. DOI: http://dx.doi.org/10.1590/S0102-311X2012000700002.

8. Gadelha CAG. Desenvolvimento e saúde: em busca de uma nova utopia. Saúde Debate. 2007 [acesso 23 Abr 2013];19(71):326-7. Disponível em: http://www.unc.br/mestrado/textos/Bibliografia-2013-Desenvolvimento-e-Saude.pdf. 
9. Mauskopf JA, Sullivan SD, Annemans L, et al. Principles of Good Practice for Budget Impact Analysis: Report of the ISPOR Task Force on Good Research Practices - Budget Impact Analysis. Value Health. 2007 [cited 2013 Apr 23];10(5):336-47. Available from: http://www.valueinhealthjournal.com/article/S1098-3015(10)60471-8/abstract? returnURL=http\%3A\%2F\%2Flinkinghub.elsevier.co m\%2Fretrieve\%2Fpii\%2FS1098301510604718\%3Fshowall\%3Dtrue. DOI: $\underline{\text { http://dx.doi.org/10.1111/j.1524-4733.2007.00187.x. }}$

10. Capucho HC, Ribeiro FC, Vidal ÁT, et al. Incorporação de Tecnologias em Saúde no Brasil: novo modelo para o Sistema Único de Saúde. BIS Bol Instituto Saúde. 2012 [acesso 23 Abr 2013];13(3):1215-22. Disponível em: http://portal.saude.gov.br/portal/arquivos/ pdf/IncorporacaodeTecnologiaemSaudenoBrasil.pdf.

11. Brasil. Conselho Federal de Medicina. Resolução 1.931, de 17 de setembro de 2009. Aprova o Código de Ética Médica. Brasília: DOU, 24 de setembro de 2009; Seção 1, p. 90. 